\title{
Modelling of Spacecraft Dynamics at Deployment of Large Elastic Structure
}

\author{
V. S. Khoroshilov ${ }^{1}$ and A. E. Zakrzhevskii ${ }^{2}$ \\ ${ }^{1}$ M. K. Yangel State Design Office "Youzhnoye", 3 Krivorozhskaja Street, Dniepropetrovsk 49008, Ukraine \\ ${ }^{2}$ S. P. Timoshenko Institute of Mechanics, NAS of Ukraine, 3 Petra Nesterova Street, Kiev 03057, Ukraine \\ Correspondence should be addressed to A. E. Zakrzhevskii, alex.zakr@mail.ru
}

Received 20 April 2012; Revised 29 June 2012; Accepted 2 July 2012

Academic Editor: Zeki Ayag

Copyright (C) 2012 V. S. Khoroshilov and A. E. Zakrzhevskii. This is an open access article distributed under the Creative Commons Attribution License, which permits unrestricted use, distribution, and reproduction in any medium, provided the original work is properly cited.

\begin{abstract}
In this paper, a new approach to the modelling of the deployment dynamics of a flexible multi-body system with the time dependent configurations is demonstrated in the frame of the study the dynamics of a spacecraft with the gyro-gravitational system of stabilization. Primarily the gravitational stabilizer that is made as a pantograph structure is in a compact form. The deployment of a flexible pantograph structure is performed after placing the spacecraft into orbit and completion of the preliminary damping by a special jet-propelled system, and after uncaging the gyros. After its deployment, the pantograph turns into an elongated structure that serves as a gravitational stabilizer and carrier of solar batteries. The objective of the study is the creation of the generalized mathematical model and the conducting of the computational modelling of the spacecraft dynamics. The equations of motion are derived with the use of the Eulerian-LaGrangian formalism and symbolic computing. Numerical simulations of the typical operational mode of the system are conducted taking into account various control profiles for the deployment. Numerical results indicate that the system used for attitude stabilization ensures the shape of the deployed design and prescribed accuracy of the orientation.
\end{abstract}

\section{Introduction}

Structures created from elements delivered into orbit in a compact condition are one of the basic components of modern space systems. Spacecraft (SC) with long flexible appendages for the exploration of the Earth's physical fields and gravitationally stabilized SC that deploys the long boom with the mass concentrated at the tip have been used since the end of the fifties of the previous century. The deployment of the space structures brings in considerable disturbances into the dynamics of the SC about its mass centre. At present, the study of such deployable structures is actually for minimization of deployment duration and power resources, for analysis of the effect of attached systems of a changeable configuration on the SC attitude. The problem of weight reduction of such systems is constantly actual. Therefore, elements of such systems should be considered as elastic bodies.

There is a large number of studies in the literature dedicated to the deployment of elastic appendages from the fixed basis as well as from the rotating SC [1-6], including the deployment of the gravity-gradient boom $[7,8]$. The maximum bending moments and the deflection of the booms at different simplifying hypothesis were evaluated in these papers; the optimization of the boom deployment timing was also fulfilled. In a relevant paper, Cherchas [9] studied the dynamics of the satellites stabilized by the rotation in the process of extension of the long flexible booms. He determined the maximum angles of nutation precession after deployment and the maximum bending moments and deflections of the rod. The equations of motion were derived by the method of Lagrange, and the elastic degrees of freedom were discredited based on the modal analysis. Lips and Modi [10], Tsuchiya [11], and Lips et al. [12] also described the dynamics of the deployed elastic elements in terms of the modal analysis with the mode shapes of natural vibrations dependent on time. In a different approach to the problem under consideration, Banerjee and Kane [6] presented a unique method for the description of beam dynamics, as it is being deployed from or retrieved 
into a rigid rotating body. The essence of the method was the modelling of the beam as a chain of elastically connected rigid links, then the method worked with the equations of motion linearized in the modal coordinates for the links located at the considered time out of the main body. Banerjee [13] and Banerjee and Nagarajan [14] extended the analysis to cases of large deflections with the order-n formulation for a variable number of bodies. The simulation results showed that the deflections of the boom tip were very sensitive to the rate of deployment/retrieval, the forcing into turned out to be less persistent process out of two ones. It was established that the character of the angular velocity of the rigid body from/into which the beam is being deployed/retrieved influences the beam behaviour.

The special position among the large structures to be deployed in space belongs to tethered spacecraft, which is connected by long thin cables, and lightweight cable nets deployable in space to serve as platforms for very large structures. One of the most important operations during a tethered satellite system mission is the deployment and retrieval of a subsatellite from or to a spacecraft. There are many studies devoted to analytical and numerical analysis of the equations of tethers motion, focused on estimation or creation of control laws of their deployment. Levin [15] and Williams [16] give the detailed analysis of basic aspects of this problem.

Only a few of the studies, which deal with the dynamics of the SC of variable configuration, take into account the dynamics of the deployment actuating mechanism. Zakrzhevskii and Khoroshilov [17] studied the dynamics of the orbital deployment of an elastic ring-shaped antenna taking into account dynamics of actuating drive. Creamer [7] studied the transverse motion of the truss boom deployed along an axis, in order to compare the theory and experiment. The boom was deployed from a canister in the horizontal plane and was supported by a bungee cord tied with the slider at the ceiling of the building in order to compensate the gravitation. Dranovskii et al. [18] stop at the development of general mathematical model of the SC during the deployment of the flexible gravitation stabilizer according to the specified programme.

Dranovskii et al. [19] studied the dynamics of SC with regard to an elastic pantograph structure deployment, but transversal displacements of the pantograph were not taken into account here. Works, except for work [19], which would investigate the dynamics of deployment in orbit of pantograph designs, are unknown to the authors. This research generalises work [19] by the additional account of transversal displacements of the deployed pantograph design with objective to study their effect on the dynamics of the SC with gyro-gravy stabilization and its elements.

\section{Problem Formulation}

2.1. Physical Model of System. Here, the SC that includes gyro-gravitational system of stabilization is studied during the deployment of a flexible pantograph structure according to a specified program into the elongated flexible gravitational stabilizer (GS) that serves also as a carrier of

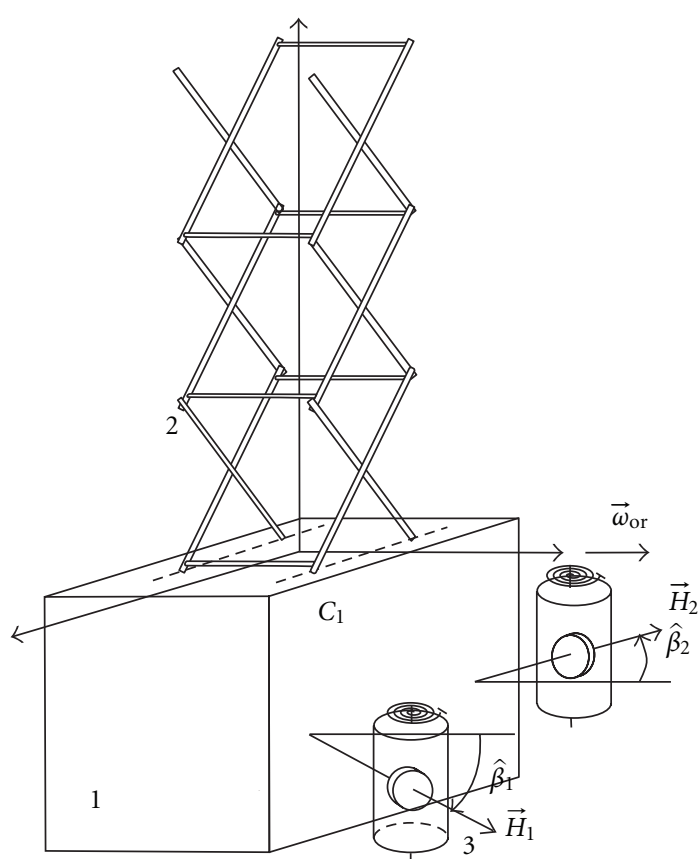

Figure 1: Basic elements of the spacecraft.

solar batteries. SC includes two gyro-dampers (GD) which are installed in order to reduce amplitudes of angular oscillations.

The SC is placed in a circular orbit about the Earth of radius $6600 \mathrm{~km}$. Basic elements of the SC under consideration are shown in Figure 1. Here, element 1 is the SC main module, 2 is the spatial structure that consist of two coupled plain pantographs, and 3 and 4 are the GD.

Figure 1 shows symbolically only three tiers of the design during deployment. Here, the structures up to 20 tiers were studied. The deployment starts when the points of joining each a plain pantograph to the main module begin to approach synchronously towards each other. The actuating drive dynamics are not taken into consideration since it is very simple and may be realized as a device that has no effect on the SC dynamics. The law of changing of the distance between the points of joining of the structure to the main module is prescribed in time.

Such deployable structures possess a considerable flexibility. Because of requirements to maintenance of the shape of the gravitational stabilizer, so that, for instance, to stabilize the spacecraft in the orbital reference frame with the predefined accuracy, some restrictions must be imposed on the deployment and design parameters. They can be determined in the process of numerical simulations. The GD role in the process of deployment and after its termination is also studied further.

2.2. Mechanical Model of System. For the case of deployment of the elastic pantograph structure in orbit, the generalized mechanical model may be represented as a main rigid body $S_{1}$ and body $S_{2}$ of variable configuration attached to it, as it is shown in Figure 2. Here, $\bar{C} \bar{X} \bar{Y} \bar{Z}$ is an earth-centred inertial 


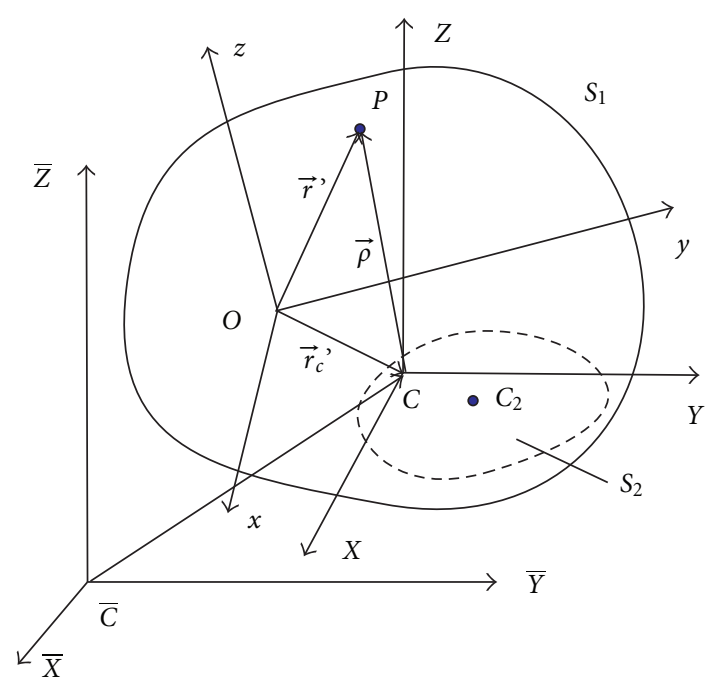

FIGURE 2: Generalized mechanical model of spacecraft with deployable designs.

reference frame with axis $\bar{C} \bar{Y}$ along the earth's spin axis, axis $\bar{C} \bar{Z}$ pointing to the vernal equinox, and axis $\bar{C} \bar{X}$ completing the orthogonal triad. $O x y z$ is a main body fixed reference frame with origin at the some point $O$ of the body $S_{1}$ with axis $\mathrm{O} z$ along the design position of the GS axis. The body $S_{1}$ is the gyro-static part; it includes the damping gyros, which do not change the rotational body inertia. The body $S_{2}$ is the deployed part with $C_{2}$ as the centre of mass and does not comprise any gyrostatic components. The body $S_{1}$ motion is defined by the velocity vector $\mathbf{v}_{O}$ of the point $O$ and vector of absolute angular velocity $\boldsymbol{\omega}$. The position vector $\mathbf{r}$ defines the location of the arbitrary point $P$ with respect to the inertial reference frame $\bar{C} \bar{X} \bar{Y} \bar{Z}$, and the position vector $\mathbf{r}^{\prime}$-with respect to the reference frame $O x y z$, fixed to the main body. In contrast to the problem of dynamics of the relative motion of the attached bodies described by Lurie [20], here one has the more general case when the expression for $\mathbf{r}^{\prime}$ depends on time $t$ explicitly, not only through the following generalized coordinates:

$$
\mathbf{r}^{\prime}=\mathbf{r}^{\prime}\left(q_{1}, \ldots, q_{n}, t\right),
$$

since the deployment of the pantograph takes place in accordance with the prescribed in time law. As a result, $\mathbf{r}^{\prime}$ changes in time during deployment even when the relative elastic motion of the structure is absent.

The additional frame of reference (Figure 3 ) can be useful also at the problem setting. The frame of reference $C x^{\text {or }} y^{\text {or }} z^{\text {or }}$ is fixed in the mass centre of the SC. This frame is introduced in such a way as in [21] with $C x^{\text {or }}$ along the SC velocity vector, $C y^{\text {or }}$ along the orbit binormal, and $C z^{\text {or }}$ along the local vertical.

In Figure 3, $\Omega$ is the longitude of the ascending node from a point of the vernal equinox, $i$ is the orbit inclination, $u$ is the argument of latitude, and $\boldsymbol{\omega}^{\text {or }}$ is the vector of the orbital angular velocity.

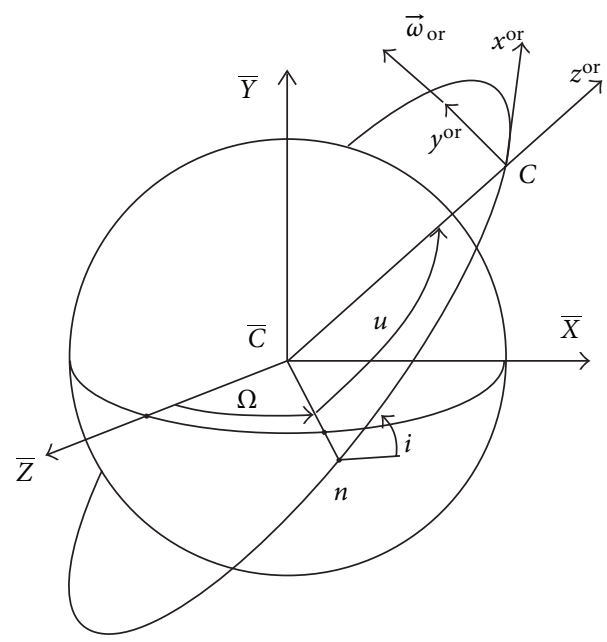

Figure 3: Coordinate frames.

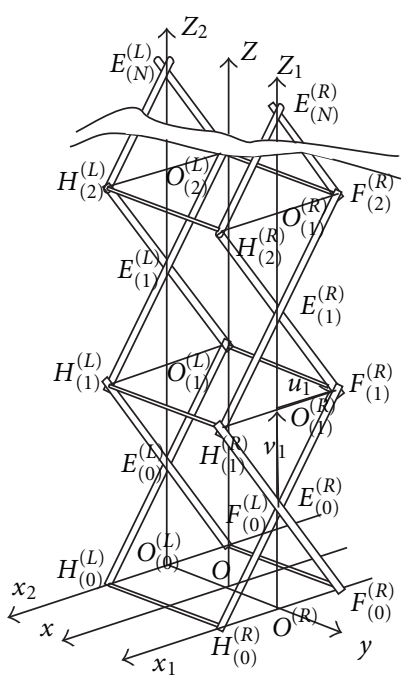

FIGURe 4: Pantograph structure.

Neglecting the orbital precession, one can consider the absolute angular velocity of the orbital frame of reference to be equal to $\boldsymbol{\omega}^{\text {or }}$.

For both pantographs, the elastic rods have their length $2 a$ and mass $2 m_{a}(i)$, where index $i$ is used for the numbering of tiers that form the full rhombuses. These rods are connected by the cylindrical hinges at the points $E_{(i)}^{(R)}, E_{(i)}^{(L)},(i=$ $\overline{0, N})$, where $N$ is the number of the tiers, and at points $H_{(i)}^{(R)}, H_{(i)}^{(L)}, F_{(i)}^{(R)}, F_{(i)}^{(L)},(i=\overline{1, N})$, where the superscripts identify the pantograph according to Figure 4.

These two pantographs are denoted by $L$ and $R$ accordingly. Thus, each pantograph consists of the zero semirhombus and the subsequent $N$ rhombuses with the sides $a$. The pantographs are connected between themselves and also out of their plane by rigid rods of the length $\tilde{b}$ and mass $m_{b}$ at points $H_{(i)}^{(R)}, H_{(i)}^{(L)}$ and $F_{(i)}^{(R)}, F_{(i)}^{(L)},(i=\overline{1, N})$, on which solar batteries are mounted. Rods of length $H_{(i)}^{(R)} H_{(i)}^{(L)}=F_{(i)}^{(R)} F_{(i)}^{(L)}=$ 


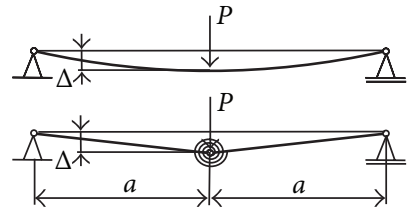

Figure 5: Beams with the same bending elasticity.

$\tilde{b},(i=\overline{1, N})$ may be considered as the third system, which is denoted further by the superscript $M$.

Change of the configuration of this structure occurs when the distance $2 b(t)$ between points $H_{0}^{(R)}, F_{0}^{(R)}$ and $H_{0}^{(L)}, F_{0}^{(L)}$ of joining each a plain pantograph with the deployment actuating drive that is established on the main body begin to decrease under the given law. The actuating drive dynamics are not taken into consideration here since this device is very simple and may be realized as a device that has no effect on the SC dynamics.

At the initial instant, the pantograph structure is in a compact form (a transport condition). The inclination angles of the rods that combine in all tiers (including the zero tier) with respect to axis $O x$ are equal to $5^{\circ}$. After the deployment termination, values of the specified angles reach $75^{\circ}$. Here, $O x y z$ is the frame of reference that is fixed in the main body with the origin in point $O$, which is located on the plane $H_{(0)}^{(R)} H_{(0)}^{(L)} F_{(0)}^{(R)} F_{(0)}^{(L)}$ at the intersection of diagonals of the given rectangle. Axes $O^{(R)} x_{1}, O x, O^{(L)} x_{2}$ and $O^{(R)} z_{1}, O z, O^{(L)} z_{2}$ are parallel.

Elastic rods of the physical model have been replaced in the mechanical model by equivalent constructions of two rigid rods connected by the spring-bias cylindrical hinges with damping. Damping is used in order to approach the dynamics to reality at least in a qualitative sense. Stiffness of the springs in the hinges is defined from the condition of equal deflections of either of the two constructions (Figure 5) for equal loads. The equivalent construction (in Figure 5 below) has the same deflection when spring resistance $c_{\text {joint }}$ in the hinge is equal to $3 E J /(2 a)$. Such a replacement is completely justified, as the configuration of the design is defined by mutual positions of the middle and end points of each rod. Besides, let the centre of each hinge have certain position and velocity that do not contradict to connections in an arbitrary state of a pantograph design. Let this state be identical for a real design and for its model. One can define for such a state of both systems their tensor of inertia, moment of momentum, and kinetic and potential energies. One can see that these values are almost identical for the real design and for the model. Differences are defined only by the small items connected with difference of local dynamic characteristics of a real bent rod in length $2 a$ and its model of two direct rods. These differences are negligible quantities as compared to these values for all design. The further complication of the model will not bring new effects. Introduction of the additional internal degrees of freedom concerned with elastic deviations in a direction of axis $O y$ is not justified, since external forces are absent in this direction.
2.3. Mathematical Model of System. The equations of motion of the system under consideration become the most compact and convenient for numerical integration (both for angular and relative motions), if one chooses the instant position of the mass centre $C$ as an origin. In this case, kinetic energy can be presented in the following form:

$$
\begin{aligned}
& T_{S_{1}+S_{2}}= \frac{1}{2} \int_{m} \mathbf{v}_{i}^{2} d m=\frac{1}{2} \int_{m}\left(\mathbf{v}_{C}+\stackrel{*}{\boldsymbol{\rho}}+\boldsymbol{\omega} \times \boldsymbol{\rho}\right)^{2} d m \\
&=\frac{1}{2} \int_{m}\left[\mathbf{v}_{C}^{2}+\stackrel{\boldsymbol{\rho}}{2}^{2}+(\boldsymbol{\omega} \times \boldsymbol{\rho})^{2}+2 \mathbf{v}_{C} \stackrel{*}{\boldsymbol{\rho}}\right. \\
&\left.+2 \mathbf{v}_{C}(\boldsymbol{\omega} \times \boldsymbol{\rho})+2 \stackrel{*}{\boldsymbol{\rho}}(\boldsymbol{\omega} \times \boldsymbol{\rho})\right] d m .
\end{aligned}
$$

Here, $\rho$ is the position vector of the system arbitrary point with respect to the instant position of the centre of mass $C$.

After integration, the expression for the kinetic energy of the whole system is given by

$$
T=\frac{M}{2} \mathbf{v}_{C}^{2}+T_{r}^{O}-\frac{M}{2} \mathbf{r}_{C}^{*^{\prime}}+\frac{1}{2} \boldsymbol{\omega} \times \boldsymbol{\Theta}^{C} \cdot \boldsymbol{\omega}+\boldsymbol{\omega} \times \mathbf{K}_{r}^{C} .
$$

The following notations are used here: $\Theta^{C}$ is the inertia tensor of the whole system with respect to point $C ; \mathbf{K}_{r}^{C}=\int_{m} \mathbf{r}^{\prime} \times \mathbf{r}^{\prime} d m-M \mathbf{r}_{C}^{\prime} \times \mathbf{r}_{C}^{\prime}$ is the relative moment of momentum of the deployed part with respect to point $C$; $\mathbf{r}_{C}^{\prime}$ is position vector of the instant position of the centre of mass $C$ in the main body fixed frame of reference; the symbol $*$ indicates the time differentiation in the main body fixed reference frame; $M$ is the total mass of the system; $\mathbf{v}_{C}$ is the absolute velocity of the centre of mass; $T_{r}^{O}$ is the kinetic energy of the relative motion of the carried bodies calculated under condition of definition of relative velocities of their points with respect to point $O$. It is easy to show that expression (3) is transformed to the more simple form if to calculate relative velocities of the specified points with respect to the instant position of the mass centre $C$ as follows:

$$
T=\frac{M}{2} \mathbf{v}_{C}^{2}+T_{r}^{C}+\frac{1}{2} \boldsymbol{\omega} \times \Theta^{C} \cdot \boldsymbol{\omega}+\boldsymbol{\omega} \times \mathbf{K}_{r}^{C} .
$$

Note that though kinetic energy of the relative motion is a scalar, it follows from the previously mentioned that it is necessary to specify a chosen origin for determination of the relative velocities for its correct calculation.

Application of the Euler's operator $E_{j}(\cdot)=$ $(d / d t)\left(\partial(\cdot) / \partial \dot{q}_{j}\right)-\partial(\cdot) / \partial q_{j}$ to the expression (3) leads to the following Lagrange's equations of the second kind for the generalized coordinates $q_{s}$ :

$$
\begin{gathered}
E_{s}\left(T_{r}^{O}\right)-M \stackrel{* *}{\mathbf{r}_{C}^{\prime}} \cdot \frac{\partial \mathbf{r}_{C}^{\prime}}{\partial q_{s}}-\frac{1}{2} \boldsymbol{\omega} \cdot \frac{\partial \Theta^{C}}{\partial q_{s}} \cdot \boldsymbol{\omega} \\
+\dot{\boldsymbol{\omega}} \cdot \frac{\partial \mathbf{K}_{r}^{C}}{\partial \dot{q}_{s}}+\boldsymbol{\omega} \cdot E_{s}^{*}\left(\mathbf{K}_{r}^{C}\right)=Q_{s},
\end{gathered}
$$

where $E_{j}^{*}(\cdot)=(\partial / \partial t)\left(\partial(\cdot) / \partial \dot{q}_{j}\right)-\partial(\cdot) / \partial q_{j}$ is also the Euler's operator, but the time differentiation is performed here in 
the reference frame $O x y z ; Q_{s}$ are the generalized forces that take into account the elastic and dissipative characteristics of the structure; $\mathbf{m}^{C}$ is the gravitational torque.

It is important to mention here that the term $E_{s}^{*}\left(\mathbf{K}_{r}^{C}\right)$ does not reduce to $(-2) \partial \mathbf{K}_{r}^{C} / \partial q_{s}$, as it is shown in [20] for $E_{s}^{*}\left(\mathbf{K}_{r}^{O}\right)$, since the case to be considered here is the more general case of nonstationary connections (see expression (1)).

The following notations are used here: $\Theta^{C}$ is the inertia tensor of the whole system with respect to the mass centre $C, \mathbf{K}_{r}^{C}=\int_{m} \mathbf{r}^{\prime} \times \stackrel{*}{\mathbf{r}^{\prime}} d m-M \mathbf{r}_{C}^{\prime} \times \mathbf{r}_{C}^{*}$ is the relative moment of momentum of the deployed part with respect to point $C$, $\mathbf{r}_{C}^{\prime}$ is the position vector of the instant position of the mass centre $C$ in the main body fixed frame of reference, and the symbol $*$ indicates the time differentiation in the main body fixed reference frame.

Euler-Lagrange equation in the vector form can be obtained simple enough if to use the moment of momentum theorem of the whole system. The total moment of momentum of the system looks like

$$
\mathbf{K}^{C}=\boldsymbol{\Theta}^{C} \cdot \boldsymbol{\omega}+\mathbf{K}_{r}^{C} .
$$

Because of application of the specified theorem, the SC attitude dynamics equation may be obtained in the following form:

$$
\boldsymbol{\Theta}^{C} \cdot \dot{\boldsymbol{\omega}}+\boldsymbol{\Theta}^{C} \cdot \boldsymbol{\omega}+\boldsymbol{\omega} \times\left(\boldsymbol{\Theta}^{C} \cdot \boldsymbol{\omega}\right)+\boldsymbol{\omega} \times \mathbf{K}_{r}^{C}=\mathbf{m}^{C},
$$

which coincides with known equation [20].

If to supplement equations (5) and (7) by kinematic equations, one obtains a closed system of equations of motion. Now, one can pose the Cauchy problem and study the dynamics of the spacecraft in time, when its geometry changes. The parameters of Rodrigues-Hamilton were chosen as kinematic parameters, which determine the attitude of the main body in the orbital reference frame in order to anticipate possible singularities in the kinematical equations of the spacecraft while conducting the simulation. Reference [17] gives details of mutual transformations between quaternion and conventional attitude angles.

Further, it is necessary to choose proper generalized co-ordinates $q_{s}$ and determine expressions for $\mathbf{r}_{c}^{\prime}\left(q_{1}, q_{2}, \ldots, q_{N}, t\right), \quad \Theta^{O}\left(q_{1}, q_{2}, \ldots, q_{N}, t\right), T_{r}^{O}\left(q_{1}, q_{2}, \ldots, q_{N}\right.$, $\left.\dot{q}_{1}, \dot{q}_{2}, \ldots, \dot{q}_{N}, t\right), \quad \mathbf{K}_{r}^{O}\left(q_{1}, q_{2}, \ldots, q_{N}, \dot{q}_{1}, \dot{q}_{2}, \ldots, \dot{q}_{N}, t\right), \quad \Pi\left(q_{1}\right.$, $\left.q_{2}, \ldots, q_{N}, t\right)$, and all their derivatives with respect to time and generalized co-ordinates, which appear in the expressions for coefficients of the governing equations (5) and (7).

Choice of the generalized co-ordinates plays the extremely important role at studying of the dynamics of complex mechanical systems. The successful choice allows to write the motion equations in the compact and transparent form and to conduct their effective integration. On the contrary, the irrational choice leads to bulky expressions for coefficients of the equations that stipulate ineffective integration (large time of calculations, necessity of significant capacities of the operative memory, accumulation of errors at evaluations, and "stiffness" of the system).

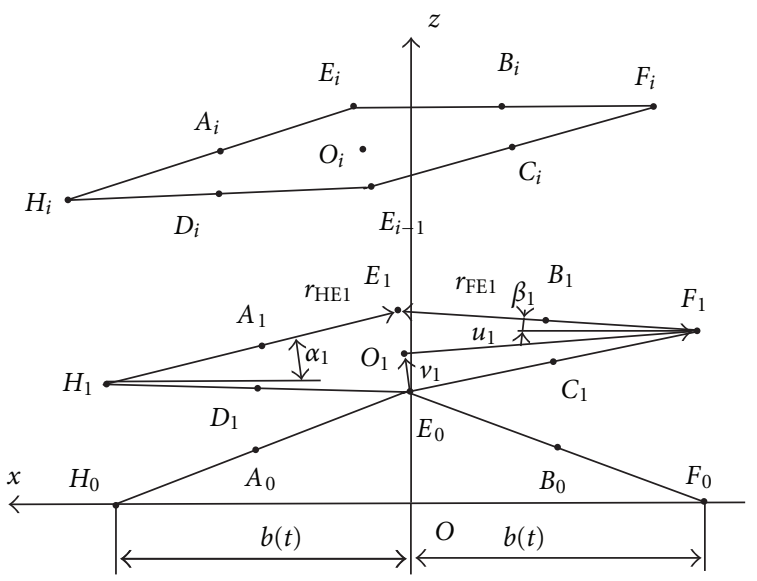

FIgURE 6: Deformations of tiers during deployment.

The SC state after its placing into orbit and completion of the preliminary damping in the orbital frame of reference, when the gyroscopes are uncaged, is considered as the initial system state.

In this case, it is impossible to consider that the design centreline remains rectilinear, as Coriolis forces act on its points during the deployment in conditions of the rotational-translational motion. These forces can produce deviations of an elastic design in the transversal direction in the orbital plane. At the same time, transversal forces are absent in the direction of the orbit binormal. Hence, the deviations in the direction of axis $O y$ can be neglected.

Values of co-ordinates $x_{i}, z_{i}(i=\overline{1, N})$ of points $E_{i}$ of each tier, lying on axis $\mathrm{Oz}$ on the straight lines connecting points $E_{(i)}^{(L)}$ and $E_{(i)}^{(R)}$ (Figure 4$)$, and angles $\tilde{\beta}_{i}(i=1,2)$ (Figure 1) have been chosen as the generalized co-ordinates in this study. Note that $z_{i}=z_{i, p}(t)+z_{i, e}$, where $z_{i, p}(t)$ are functions of time, which are determined by the deployment time schedule, and $z_{i, e}$ are independent variables.

Using Figure 4, it is simple to show the position vector of the mass centre of $i$ th tier of the pantograph design is

$$
\mathbf{r}_{O}^{\prime}(i)=\left\{\frac{x(-1+i)+x(i)}{2}, 0, \frac{(z(-1+i)+z(i))}{2}\right\}
$$

As a result, for SC as a whole,

$$
\begin{gathered}
\mathbf{r}_{O}^{\prime}=\left(\frac{\left\{a_{x}, a_{y}, a_{z}\right\} m_{K A}+\left\{0,0, \tilde{z}_{0}\right\}\left(m_{0}+m_{1}\right)}{M}\right. \\
\left.+\frac{\sum_{i=1}^{N}\left\{x_{i}, 0, z_{i}\right\} \tilde{m}_{i}}{M}\right) .
\end{gathered}
$$

Here, $\left\{a_{x}, a_{y}, a_{z}\right\}$ is the position vector of the mass centre of the main body in the frame of reference $O x y z ; m_{K A}$ is mass of the main body; $m_{i}(i=\overline{0, N})$ is mass of $i$ th tier; $\left\{x_{i}, 0, z_{i}\right\}$ is the position vectors of points $E_{i} ; \tilde{m}_{i}=\left(m_{i}+m_{i+1}\right) / 2, \tilde{m}_{N}=$ $m_{N} / 2, \widetilde{z}_{0}=z_{0} k_{0} ; k_{0}$ is the coefficient defining the position of the mass centre of the zero tier on axis $O z ; b(t)=H_{0} O=O F_{0}$ is known function of time which determines the law of the design deployment. 
2.4. Main Dynamical Values. The inertia tensor of the pantograph design can be written in the form of the sum of the local tensors of inertia of single rods in the frame of reference that is parallel to axis $O x y z$ with the origin in the mass centre of corresponding rods, and the tensor of inertia of all point masses disposed in the centres of mass of the rods that are equal to masses of these rods. It gives the following expressions for $i$ th tier:

$$
\begin{aligned}
& \Theta^{O}{ }_{1,1, i}=2 J_{i}^{M}+\frac{\left(a^{2} m_{i} \sin ^{2} \alpha_{i}\right)}{3}+\frac{\left(a^{2} m_{i} \sin ^{2} \beta_{i}\right)}{3}+\frac{\left(m_{i}^{M} d z_{i}^{2}\right)}{2} \\
& +\frac{\left(2 m_{i}^{M} d x_{i}^{2} u_{i}^{2}\right)}{L_{i}^{2}}-2 m_{i}^{M} d z_{i} z_{i}+2 m_{i}^{M} z_{i}^{2} \\
& +m_{i}\left(\frac{5 d z_{i}^{2}}{2}+\frac{2 d x_{i}^{2} u_{i}^{2}}{e L 1_{i}^{2}}-8 d z_{i} z_{i}+8\left(b b^{2}+z_{i}^{2}\right)\right), \\
& \Theta_{1,3, i}^{O}=\frac{\left(a^{2} m_{i}\left(\sin \left(2 \alpha_{i}\right)+\sin \left(2 \beta_{i}\right)\right)\right)}{6} \\
& +m_{i}^{M}\left(x_{i}\left(d z_{i}-2 z_{i}\right)+d x_{i}\left(d z_{i}\left(-0.5+\frac{2 u_{i}^{2}}{L_{i}^{2}}\right)+z_{i}\right)\right) \\
& +m_{i}\left(4 x_{i}\left(d z_{i}-2 z_{i}\right)+d x_{i}\left(d z_{i}\left(-2.5+\frac{2 u_{i}^{2}}{L_{i}^{2}}\right)+4 z_{i}\right)\right), \\
& \Theta_{2,2, i}^{O}=\left(16 J_{i} L_{i}^{2}+m_{i}^{M}\left(d x_{i}^{2}\left(L_{i}^{2}+4 u_{i}^{2}\right)\right.\right. \\
& +d z_{i}^{2}\left(L_{i}^{2}+4 u_{i}^{2}\right)-4 d x_{i} L_{i}^{2} x_{i} \\
& \left.-4 d z_{i} L_{i}^{2} z_{i}+4 L_{i}^{2}\left(x_{i}^{2}+z_{i}^{2}\right)\right) \\
& +m_{i}\left(d x_{i}^{2}\left(5 L_{i}^{2}+4 u_{i}^{2}\right)+d z_{i}^{2}\left(5 L_{i}^{2}+4 u_{i}^{2}\right)\right. \\
& -16 d x_{i} L_{i}^{2} x_{i}-16 d z_{i} L_{i}^{2} z_{i} \\
& \left.\left.+16 L_{i}^{2}\left(x_{i}^{2}+z_{i}^{2}\right)\right)\right) /\left(2 L_{i}^{2}\right), \\
& \Theta_{3,3, i}^{O}=2 J_{i}^{M}+\frac{\left(a^{2} m_{i} \cos ^{2} \alpha_{i}\right)}{3}+\frac{a^{2} m_{i} \cos ^{2} \beta_{i}}{3} \\
& +\frac{m_{i}^{M} d x_{i}^{2}}{2}+\frac{2 m_{i}^{M} d z_{i}^{2} u_{i}^{2}}{L_{i}^{2}}-2 m_{i}^{M} d x_{i} x_{i}+2 m_{i}^{M} x_{i}^{2} \\
& +m_{i}\left(\frac{5 d x_{i}^{2}}{2}+\frac{2 d z_{i}^{2} u_{i}^{2}}{L_{i}^{2}}-8 d x_{i} x_{i}+8\left(b b^{2}+x_{i}^{2}\right)\right), \\
& \Theta_{1,1,0}^{O}=2 J_{0}^{M}+4 m_{0} b b^{2}-m_{0} b^{2}+m_{0} a^{2}+2 m_{0}^{M} z_{0}^{2} \\
& +\frac{m_{0} a^{2} \sin ^{2} \alpha_{0}}{6}+\frac{m_{0} a^{2} \sin ^{2} \beta_{0}}{6} \\
& \Theta_{1,3,0}^{O}=\frac{\left(m_{0} a^{2}\left(\sin \left(2 \alpha_{0}\right)+\sin \left(2 \beta_{0}\right)\right)\right)}{12}, \\
& \Theta_{2,2,0}^{O}=4 J_{0}+2 m_{0}^{M} b^{2}+m_{0} a^{2}+2 m_{0}^{M} z_{0}^{2},
\end{aligned}
$$

$$
\begin{aligned}
\Theta_{3,3,0}^{O}= & \left(m_{0}+2 m_{0}^{M}\right) b^{2} \\
& +\frac{\left(12 J_{0}^{M}+24 m_{0} b b^{2}+m_{0} a^{2} \cos ^{2} \alpha_{0}+m_{0} a^{2} \cos ^{2} \beta_{0}\right)}{6},
\end{aligned}
$$

where $\Theta_{k, j, i}^{O},(k, j=1,2,3)$ are $(k, j)$ component of the inertia tensor for $i$ th tier; $m_{i}$ is mass of one rod in the rhombus; $m_{i}^{M}$ is mass of one connecting rod; $J_{i}$ is proper moment of inertia of the rod with respect to its principal central axis that is parallel to axis $O y ; J_{i}^{M}$ is the characteristic moment of inertia of the connecting rod with respect to its principal central axis that is parallel to axis $O x ; d x_{i}=x_{i}-x_{i-1} ; d z_{i}=z_{i}-z_{i-1}$; $u_{i}$ is length of the line segment $H_{i} O_{i} ; L_{i}$ is length of $E_{i} E_{i-1}$; angles of inclination of rods to axis $O x \alpha_{i}$ and $\beta_{i}$ are shown in Figure 6 for the first tier; index $i$ shows that the given value concerns to $i$ th tier.

The expression for the kinetic energy of relative motion may be presented as follows:

$$
\begin{aligned}
& \operatorname{Tr}_{i}^{O}=\left(128 J_{i}\left(\stackrel{*}{\alpha_{i}^{2}}+\stackrel{*}{\beta_{i}^{2}}\right) L_{i}^{6} u_{i}^{2}\right. \\
& +m_{i}^{M}\left(16 a^{2} d x_{i}^{2} d x z 1 t_{i}^{2}\right. \\
& +16 a^{2} d x z 1 t_{i}^{2} d z_{i}^{2} \\
& -64 a^{2} d x_{i} d x 1 t_{i} d x z 1 t_{i} L_{i}^{2} u_{i}^{2} \\
& -64 a^{2} d x z 1 t_{i} d z_{i} d z 1 t_{i} L_{i}^{2} u_{i}^{2} \\
& +16 L_{i}^{4} u_{i}^{2}\left(4 a^{2} d x 1 t_{i}^{2}+4 a^{2} d z 1 t_{i}^{2}\right. \\
& -4 d x 1 t_{i} L_{i}^{2} \times 1 t_{i} \\
& -4 d z 1 t_{i} L_{i}^{2} z 1 t_{i} \\
& \left.\left.+4 L_{i}^{2}\left(x 1 t_{i}^{2}+z 1 t_{i}^{2}\right)\right)\right) \\
& +m_{i}\left(16 a^{2} d x_{i}^{2} d x z 1 t_{i}^{2}+16 a^{2} d x z 1 t_{i}^{2} d z_{i}^{2}\right. \\
& -64 a^{2} d x_{i} d x 1 t_{i} d x z 1 t_{i} L_{i}^{2} u_{i}^{2} \\
& -64 a^{2} d x z 1 t_{i} d z_{i} d z 1 t_{i} L_{i}^{2} u_{i}^{2}+16 L_{i}^{4} u_{i}^{2} \\
& \times\left(d x 1 t_{i}^{2}\left(5 L_{i}^{2}+4 u_{i}^{2}\right)\right. \\
& +d z 1 t_{i}^{2}\left(5 L_{i}^{2}+4 u_{i}^{2}\right)-16 d x 1 t_{i} L_{i}^{2} x 1 t_{i} \\
& -16 d z 1 t_{i} L_{i}^{2} z 1 t_{i} \\
& \left.\left.\left.+16 L_{i}^{2}\left(x 1 t_{i}^{2}+z 1 t_{i}^{2}\right)\right)\right)\right) /\left(64 L_{i}^{6} u_{i}^{2}\right) \text {. }
\end{aligned}
$$

For the gyro-dampers:

$$
\begin{array}{r}
T_{r}^{\left(\mathrm{gd}_{i}\right)}=\vec{\omega}_{\mathrm{rot}_{i}} \cdot \Theta_{\mathrm{rot}_{i}}^{O_{i}} \cdot \frac{\vec{\omega}_{\mathrm{rot}_{i}}}{2}+\vec{\omega}_{c s_{i}} \cdot \Theta_{c s_{i}}^{O_{i}} \cdot \frac{\vec{\omega}_{c s_{i}}}{2}, \\
(i=1,2) .
\end{array}
$$


Here, the following notations are used additionally: $T r_{i}^{O}$ is the kinetic energy of the relative motion of $i$ th tier when the relative velocities are evaluated in the frame of reference $O x y z ; x 1 t_{i}=\partial x_{i} / \partial t ; z 1 t_{i}=\partial z_{i} / \partial t ; d x 1 t_{i}=\partial\left(d x_{i}\right) / \partial t ;$ $d z 1 t_{i}=\partial\left(d z_{i}\right) / \partial t ; d x z 1 t_{i}=2\left(d x_{i} d x 1 t_{i}+d z_{i} d z 1 t_{i}\right) ; i$ is the tier number; $\vec{\omega}_{\text {rot }_{i}}=\left\{\dot{\beta}_{i}, \Omega_{\text {rot }_{i}}, 0\right\} ; \vec{\omega}_{c s_{i}}=\left\{\dot{\beta}_{i}, 0,0\right\}$ in the GD reference frames.

Substituting all used notations into (11) and (12), one may apply the Eulerian operator to such expressions with respect to particular generalized co-ordinates taking into consideration the fact that the generalized co-ordinate and a velocity with index $i$ are included into the full expression for $T_{r}^{O}$ for the tiers $i$ and tier $i-1$ (except the first tier since motion of elements of the zero tier depends explicitly on time but not on generalized co-ordinates).

Expression for projections of the angular momentum into axis $O y$ for $i$ th tier has been reduced to the following form:

$$
\begin{gathered}
K r_{i}^{O}=\frac{\left(8 J_{i}\left(d x 1 t_{i} d z_{i}-d x_{i} d z 1 t_{i}\right)\right)}{L_{i}^{2}} \\
+\left(m _ { i } \left(x 1 t _ { i } \left(-d z_{i}^{3}+\left(-4 a^{2}+4 d x_{i}^{2}+3 d z_{i}^{2}\right) z_{i-1}\right.\right.\right. \\
\left.+4\left(a^{2}+d x_{i}^{2}-5 d z_{i}^{2}\right) z_{i}\right) \\
+x 1 t_{i-1}\left(d z_{i}^{3}+\left(4 a^{2}+4 d x_{i}^{2}+5 d z_{i}^{2}\right) z_{i-1}\right. \\
\left.+\left(-4 a^{2}+4 d x_{i}^{2}+3 d z_{i}^{2}\right) z_{i}\right) \\
+\left(d x_{i}^{3}+\left(4 a^{2}+5 d x_{i}^{2}+4 d z_{i}^{2}\right) x_{i-1}\right. \\
\left.+\left(-4 a^{2}+3 d x_{i}^{2}+4 d z_{i}^{2}\right) x_{i}\right) z 1 t_{i-1} \\
+\left(d x_{i}^{3}+\left(4 a^{2}-3 d x_{i}^{2}-4 d z_{i}^{2}\right) x_{i-1}\right. \\
\left.\left.\left.-\left(4 a^{2}+5 d x_{i}^{2}+4 d z_{i}^{2}\right) x_{i}\right) z 1 t_{i}\right)\right) /\left(2 L_{i}^{2}\right) \\
+\left(m _ { i } ^ { M } \left(x 1 t _ { i } \left(-d z_{i}^{3}+\left(-4 a^{2}+d x_{i}^{2}+L_{i}^{2}\right) z_{i-1}\right.\right.\right. \\
\left.+\left(4 a^{2}-d x_{i}^{2}+L_{i}^{2}\right) z_{i}\right) \\
+x 1 t_{i-1}\left(d z_{i}^{3}+\left(4 a^{2}-d x_{i}^{2}+L_{i}^{2}\right) z_{i-1}\right. \\
\left.+\left(-4 a^{2}+d x_{i}^{2}+L_{i}^{2}\right) z_{i}\right) \\
+\left(d x_{i}^{3}+\left(4 a^{2}-d z_{i}^{2}-L_{i}^{2}\right) x_{i-1}\right. \\
+\left(4 a^{2}-d z_{i}^{2}+L_{i}^{2}\right) x_{i-1} \\
\left.\left.\left.\left.+\left(-4 a^{2}+d z_{i}^{2}+L_{i}^{2}\right) x_{i}\right) z 1 t_{i-1}\right) z 1 t_{i}\right)\right) /\left(2 L_{i}^{2}\right) . \\
+(-1)
\end{gathered}
$$

Two remaining components of $\mathbf{K}_{r}^{O}$ are equal to zero in the considered problem as all relative motions happen in the planes that are parallel to the plane $O x z$.

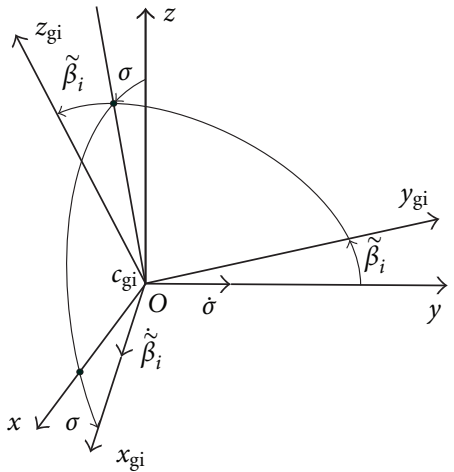

FIGURE 7: Sequence of rotations for GD axes.

For ith gyro-damper $\vec{K}_{r}^{O}(i)=\mathrm{T}_{c-\text { gd }}(i)\left\{J_{c s} \dot{\widetilde{\beta}}_{i}, H_{g}, 0\right\}(i=$ $1,2)$. The transformation matrix $\mathbf{T}_{c_{-} \text {gd }}(i)$ has the following form:

$$
\left|\begin{array}{ccc}
\cos \sigma & \sin \left(\tilde{\beta}_{i}+\tilde{\beta}_{i 0}\right) \sin \sigma & \cos \left(\tilde{\beta}_{i}+\widetilde{\beta}_{i 0}\right) \sin \sigma \\
0 & \cos \left(\widetilde{\beta}_{i}+\widetilde{\beta}_{i 0}\right) & -\sin \left(\widetilde{\beta}_{i}+\widetilde{\beta}_{i 0}\right) \\
-\sin \sigma & \cos \sigma \sin \left(\widetilde{\beta}_{i}+\widetilde{\beta}_{i 0}\right) & \cos \left(\widetilde{\beta}_{i}+\widetilde{\beta}_{i 0}\right) \cos \sigma
\end{array}\right| .
$$

It is defined by the sequence of rotations of the frames of reference fixed to the GD cases around the axes Oxyz (see Figure 7).

The potential energy of elastic displacements of the pantograph structure for $i$ th tier looks like

$$
\begin{aligned}
\Pi_{i}=c_{\text {joint }}[ & \left(\alpha_{i}-\alpha_{i-1}\right)^{2}+\left(\alpha_{i+1}-\alpha_{i}\right)^{2}+\left(\alpha_{i+2}-\alpha_{i+1}\right)^{2} \\
& \left.+\left(\beta_{i}-\beta_{i-1}\right)^{2}+\left(\beta_{i+1}-\beta_{i}\right)^{2}+\left(\beta_{i+2}-\beta_{i+1}\right)^{2}\right] .
\end{aligned}
$$

Here, $\alpha$ and $\beta$ with subscripts are angles that are shown in Figure 6. Naturally, this expression must be corrected for two last tiers.

The generalized forces of flexible displacements may be determined as $Q x_{i}=-\partial \Pi_{i} / \partial x_{i}, Q z_{i}=-\partial \Pi_{i} / \partial z_{i}$.

Except the generalized forces of elastic displacements, the design is subjected to the forces of Coulomb friction in its real hinges and damping torques in the fictitious hinges, which have appeared because of finite-element modelling of elastic rods according to Figure 5. There are six real hinges (four side hinges and two central ones) in each tier. For simplicity, it is possible to consider that moments of a Coulomb friction in such hinges are identical, as exact quantitative estimations cannot be determined. Actually, these moments are dependent on time at longitudinal oscillations of the design. If to write the virtual work for all real hinges of the design as $\delta^{\prime} A_{i}=6 M_{\text {Coul }}\left(\delta \alpha_{i}+\delta \beta_{i}\right) \operatorname{sign}\left(\dot{\alpha}_{i}+\dot{\beta}_{i}\right)$ and select coefficients at variations of generalized co-ordinates in the expression of the total virtual work, one can obtain the expressions for the generalized forces corresponding to the Coulomb friction.

The information on structural damping hardly can be enough exacted, nevertheless a qualitative evaluation needs 
to be spent. Considering that the fictitious hinge in the equivalent system (in Figure 5 below) includes not only a spring but also a viscous damper that corresponds to Kelvin-Voigt model, it is possible to write expressions for a dissipative function of four fictitious hinges of each a tier as follows:

$$
\begin{aligned}
R_{i}=r_{\text {joint }} & {\left[\left(\dot{\alpha}_{i}-\dot{\alpha}_{i-1}\right)^{2}+\left(\dot{\alpha}_{i+1}-\dot{\alpha}_{i}\right)^{2}+\left(\dot{\alpha}_{i+2}-\dot{\alpha}_{i+1}\right)^{2}\right.} \\
& \left.+\left(\dot{\beta}_{i}-\dot{\beta}_{i-1}\right)^{2}+\left(\dot{\beta}_{i+1}-\dot{\beta}_{i}\right)^{2}+\left(\dot{\beta}_{i+2}-\dot{\beta}_{i+1}\right)^{2}\right] .
\end{aligned}
$$

The approximate value of the dissipative parameter $r_{\text {joint }}$ can be defined from the Lagrange equation for a separate equivalent design, if to reduce it to its classical form $\ddot{\Delta}+2 n \dot{\Delta}+\omega_{0}^{2} \Delta=0$, where $n=3 r_{\text {joint }} /\left(m a^{2}\right)$. Taking into account the expression for the defined above spring stiffness in the hinge, one can obtain the expression $r_{\text {joint }}=$ $\delta a \sqrt{2 c_{\text {joint }} m / 3\left(4 \pi^{2}-\delta^{2}\right)} \approx(\delta a / 2 \pi) \sqrt{2 c_{\text {joint }} m / 3}$. Here $\delta$ is a decrement of oscillations. Its value can be accepted such, as for steel designs. As a result, it is possible to obtain the expressions for the generalized forces of the structural damping in the fictitious hinges in the following form: $Q R x_{i}=\partial \sum_{j} R_{j} / \partial x_{i}, Q R z_{i}=\partial \sum_{j} R_{j} / \partial z_{i}$. Corresponding analytical expressions for all main dynamic values have been obtained in Mathematica 5. form:

The potential energy of the GD springs has the following

$$
\Pi_{\mathrm{GD}}=\frac{\left(\tilde{\beta}_{1}-\tilde{\beta}_{01}\right)^{2}}{2} k_{4 \beta_{1}}+\frac{\left(\tilde{\beta}_{2}-\tilde{\beta}_{02}\right)^{2}}{2} k_{4 \beta_{2}},
$$

where $k_{4 \beta i}(i=1,2)$ is the GD springs resistance, and $\widetilde{\beta}_{0 i}(i=$ $1,2)$ are the values that $\widetilde{\beta}_{i}$ possess, if the frame of reference $O x y z$ does not rotate in the inertial basis.

For definition of coefficients of the motion equations (5) and (7), the obtained vector and tensor expressions and all their derivatives should be transformed to the frame of reference that is fixed in an instant position of the mass centre with its axes parallel to the $O x y z$ axes with the same names.

According to [20], it is possible to write

$$
\begin{gathered}
\Theta^{C}=\Theta^{O}-M \cdot\left(E r_{c}^{\prime 2}-\overrightarrow{r_{c}^{\prime}} \overrightarrow{r_{c}^{\prime}}\right), \\
\vec{K}_{r}^{C}=\vec{K}_{r}^{O}-M \cdot \overrightarrow{r_{c}^{\prime}} \times \overrightarrow{r_{c}^{\prime}}, \\
\Theta^{*}=\Theta^{*}-M\left(2 E \overrightarrow{r_{c}^{\prime}} \overrightarrow{r_{c}^{\prime}}-\overrightarrow{r_{c}^{\prime}} \overrightarrow{r^{\prime}}-\overrightarrow{r^{\prime}} \overrightarrow{r_{c}^{\prime}}\right), \\
\frac{\partial \Theta^{C}}{\partial q_{s}}=\frac{\partial \Theta^{O}}{\partial q_{s}}-2 M E\left(\overrightarrow{r_{c}^{\prime}} \cdot \frac{\partial \overrightarrow{r_{c}^{\prime}}}{\partial q_{s}}\right)+2 M\left(\overrightarrow{r_{c}^{\prime}} \frac{\partial \overrightarrow{r_{c}^{\prime}}}{\partial q_{s}}\right), \\
\vec{K}_{r}^{C}=\vec{K}_{r}^{O}-M \overrightarrow{r_{c}^{\prime}} \times \overrightarrow{r_{c}^{\prime}} .
\end{gathered}
$$

Here, $E$ is the identity matrix; the expressions $\vec{a} \cdot \vec{b}$ and $\vec{a} \times \vec{b}$ mean scalar and vector products of vectors accordingly; the expression $\vec{a} \vec{b}$ means a dyadic product.
Having substituted the obtained expressions in (5) and (7) after completing them with suitable kinematic equations, one can formulate the Cauchy problem for study the of dynamics of the system under consideration during its deployment.

\section{Simulation}

For numerical integration, the system is written in the following implicit form:

$$
\begin{aligned}
& \sum_{j=1}^{N_{\mathrm{eq}}} A_{i j}\left(y_{1}, y_{2}, \ldots, y_{N_{\mathrm{eq}}}, t\right) \dot{y}_{j} \\
& \quad=f_{i}\left(y_{1}, y_{2}, \ldots, y_{N_{\mathrm{eq}}}, t\right) \quad\left(i=\overline{1, N_{\mathrm{eq}}}\right) .
\end{aligned}
$$

In the case under consideration, $y_{i}=\omega_{i}(i=1,2,3)$. If the problem is written in the quaternions, $y_{i}(i=\overline{4,7})$ define the quaternion components. If the problem is written in the Bryan angles, then the same variables define the angles (in this case $\left.y_{7} \equiv 0\right)$. Remaining variables are the generalized coordinates and their first derivatives with respect to time, that is,

$$
\begin{gathered}
y_{4+4 i}=x_{i}, \quad y_{5+4 i}=x 1 t_{i}, \\
y_{6+4 i}=z_{i}, \quad y_{7+4 i}=z 1 t_{i} \quad(i=\overline{1, N}), \\
y_{8+4 N}=\tilde{\beta}_{1}, \quad y_{9+4 N}=\dot{\tilde{\beta}}_{1}, \\
y_{10+4 N}=\tilde{\beta}_{2}, \quad y_{11+4 N}=\dot{\tilde{\beta}}_{2} .
\end{gathered}
$$

So, the total number of variables is $N_{\mathrm{eq}}=11+4 N$. The original computation package is developed for numerical integration of the ordinary differential equations in the frame of the Cauchy problem. The majority of operators of the program is obtained in the form of Fortran expressions in Mathematica 5 on the basis of realization of some packages written especially for the studied system, and after replacement of the obtained bulky expressions with simple enough variables.

The pantograph structures having 5, 10, and 20 tiers were studied. The Cauchy problem integration step was chosen from a condition of stability of the computation process on a studied interval of time. Its magnitude varied in the range of 0.01-0.001 s. Initial values of components of $\boldsymbol{\omega}$ differ from the components of orbital angular velocity on $\pm 0.0001 \mathrm{~s}^{-1}$.

It is obvious that the dynamics of a complex flexible structure depend on the time history of the deployment (low of the deployment). Two such time histories were used here. The deployment of space designs often used such a law $b(t)$ that the velocity and acceleration time history of an actuating motor $\dot{b}(t)$ looks like the line with the mark "stand" in Figure 8. Such a law is used conventionally at the deployment of such space structures as solar batteries. Such a function $\dot{b}(t)$ stand has two angular points, and function $\ddot{b}(t)$ stand (and a function of an actuating force or torque) has four points of discontinuities. Action of such a law on 


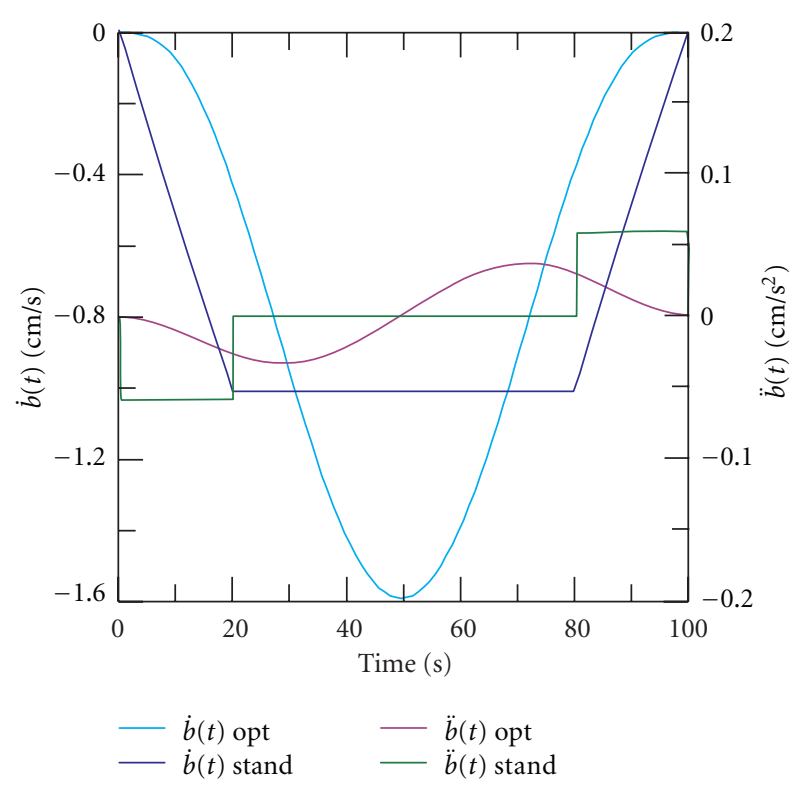

Figure 8: Laws of deployment.

an oscillating system brings additional perturbations in its dynamics.

Therefore, the alternative law of deployment of the design was considered. For this law, $\ddot{b}(t)$ does not contain points of discontinuities. Such a law can be constructed as a solution of the optimal control problem of rest-to-rest transfer of a mechanical system with internal oscillating degrees of freedom at an integral minimisation of relative accelerations of the generalised co-ordinates (see [22], Special Case II). The velocity time history of an actuating motor $\dot{b}(t)$ opt and $\ddot{b}(t)$ opt for the second law looks like the line 2 in Figure 8.

The orbit inclination $i$ is assumed to be 1 , the longitude $\Omega$ of the ascending node is taken to be zero. The moment of the central Newtonian field, corresponding to a circular orbit of $600 \mathrm{~km}$ altitude, was considered as the external perturbing moment. Though the SC movement along an orbit is not considered here, the orbit parameters are required to calculate the gravitational torque and projections of the total SC moment of momentum to the absolute frame of reference. It is necessary for the monitoring of errors of the numerical integration of the initial value problem.

Key system parameters are shown in the Table 1.

A series of evaluations have been conducted at different values of system parameters in the process of the numerical simulation of the deployment dynamics of the pantograph design. The attitude modification of the SC main body under the effect of the structure deployment and dynamics of a modification of the shape of the construction depending on the adopted parameters were studied.

Design deployment essentially increases the components of the inertia tensor $\Theta_{1,1}^{C}$ and $\Theta_{2,2}^{C}$ and decreases slightly the component $\Theta_{3,3}^{C}$. Figure 9 shows the time history of the diagonal components of the total inertia tensor of SC for both standard and optimal laws of deployment. Here, one can see that $\Theta_{1,1}$ and $\Theta_{2,2}$, which differ at the
TABLE 1: Key system parameters.

\begin{tabular}{lcc}
\hline Parameter & Notations & Value \\
\hline Mass of main body & $m_{1}$ & $350 \mathrm{~kg}$ \\
Rods mass & $m_{l}$ & $1 \mathrm{~kg}$ \\
Rods bending stiffness & $E J$ & $20-80 \mathrm{~N} \mathrm{~m}^{2}$ \\
Decrement of oscillations & $\vartheta$ & 0.001 \\
Nonzero components of the main body & $J_{x x}$ & $4000 \mathrm{~kg} \mathrm{~m}^{2}$ \\
inertia tensor & $J_{y y}$ & $5000 \mathrm{~kg} \mathrm{~m}^{2}$ \\
& $J_{z z}$ & $2000 \mathrm{~kg} \mathrm{~m}^{2}$ \\
& $a_{x}$ & $0 \mathrm{~m}$ \\
Shift of main body centre of mass & $a_{y}$ & $0 \mathrm{~m}$ \\
& $a_{z}$ & $-1 \mathrm{~m}$ \\
Angular momentum of one GD rotor & $h_{r o t}$ & $20 \mathrm{~kg} \mathrm{~m} / \mathrm{s}$ \\
GD damping coefficient & $k_{3 \beta_{1}, \beta_{2}}$ & $40 \mathrm{~N} \mathrm{~m} / \mathrm{s}^{2}$ \\
Duration of deployment & $T_{f}$ & $100-1000 \mathrm{~s}$ \\
\hline
\end{tabular}

beginning, then behave practically synchronously with oscillations of the pantograph length. Component $\Theta_{3,3}$ decreases as the distances of pantograph's points to axis $\mathrm{Oz}$ decrease noticeably during the deployment. One can see that oscillation amplitudes of the pantograph length are essentially less when optimum laws are used. Generally speaking, the inertia tensor is not diagonal one in the presence of transversal design deviations in the direction of axis $O x$. The nonzero component $\Theta_{1,3}$ appears at that, but in view of smallness of these deviations as compared to the longitudinal displacements, it is negligibly small also as compared to the diagonal components of the inertia tensor and has no essential effect on the SC dynamics.

The increase in the inertia tensor components leads to sharp decrease in the component $\omega_{2}$ of the SC angular velocity vector (Figure 10) and naturally to loss of the SC orientation in the orbital frame of reference. At the same time, generalized co-ordinates $z_{i}(i=4,8,12,16,20)$ behave according to Figure 11. Such behaviour speaks about appreciable longitudinal oscillations of the design at the deployment stage. These oscillating components are induced by excitation of elastic oscillations of design rods. Their amplitudes grow with increase of number of the tier. Longitudinal oscillations have noticeable influence upon the components of the inertia tensor (Figure 9). Behaviour of angles of the rods tilt $\alpha_{i}$ with the same values of the subscripts is shown in Figure 12. Here, one can see that in the beginning of the deployment, magnitudes of the angles become less than their initial values under the effect of forces of rest inertia. As far as the relative acceleration, which the deploying mechanism tries to impose to the upper tiers, is greater than that for the lower ones, their "draw" is more appreciable.

The transversal relative deviations of the design longitudinal axis defined by the generalized co-ordinates $x_{i}(i=$ $4,8,12,16,20)$ are shown in Figure 13. Practically, all these deviations are directed opposite to the vector of the SC 


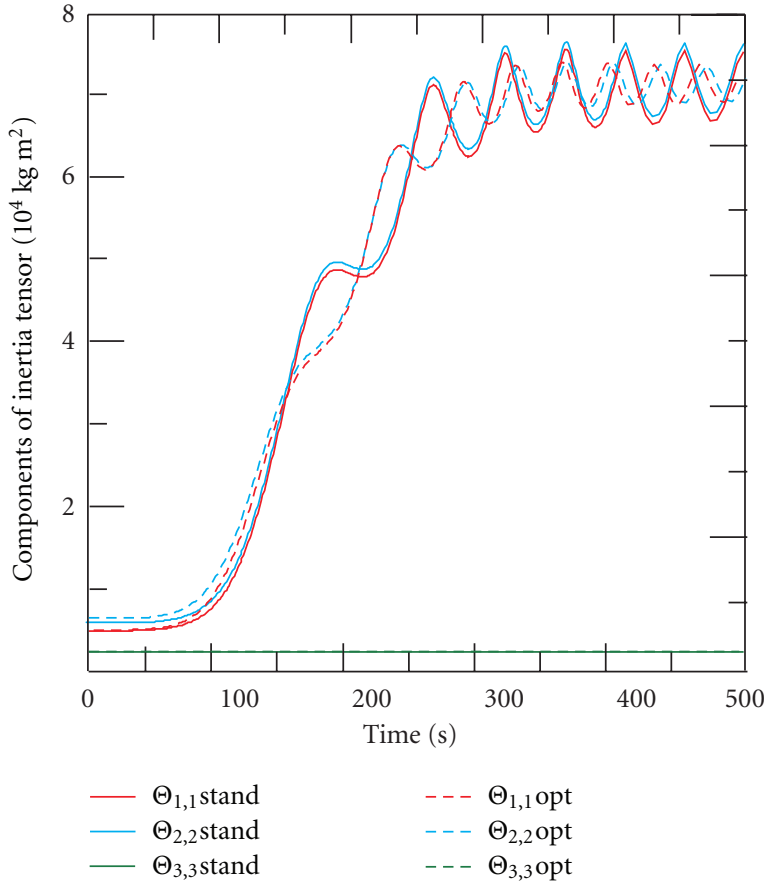

Figure 9: Time dependence of inertia tensor diagonal components.

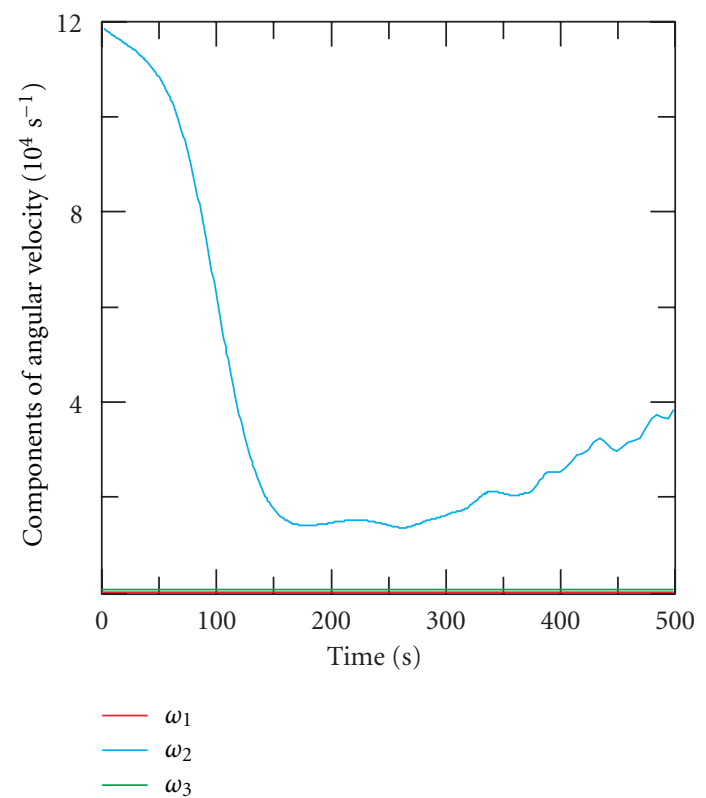

FIGURE 10: Time histories of absolute angular velocity projections.

velocity under the influence of the Coriolis forces. Figures $9,10,11,12$, and 13 correspond to the standard law of deployment. If to use the optimal law mentioned above, at the same values of the remaining parameters, the amplitudes of oscillations $z_{i}$ are essentially smaller than what one can see in Figure 14.

One of the considered variants has allowed to analyze the dynamics of the system at the same flexural rigidity, but at twice as long deployment, that is, at $T_{f}=1000 \mathrm{~s}$. The

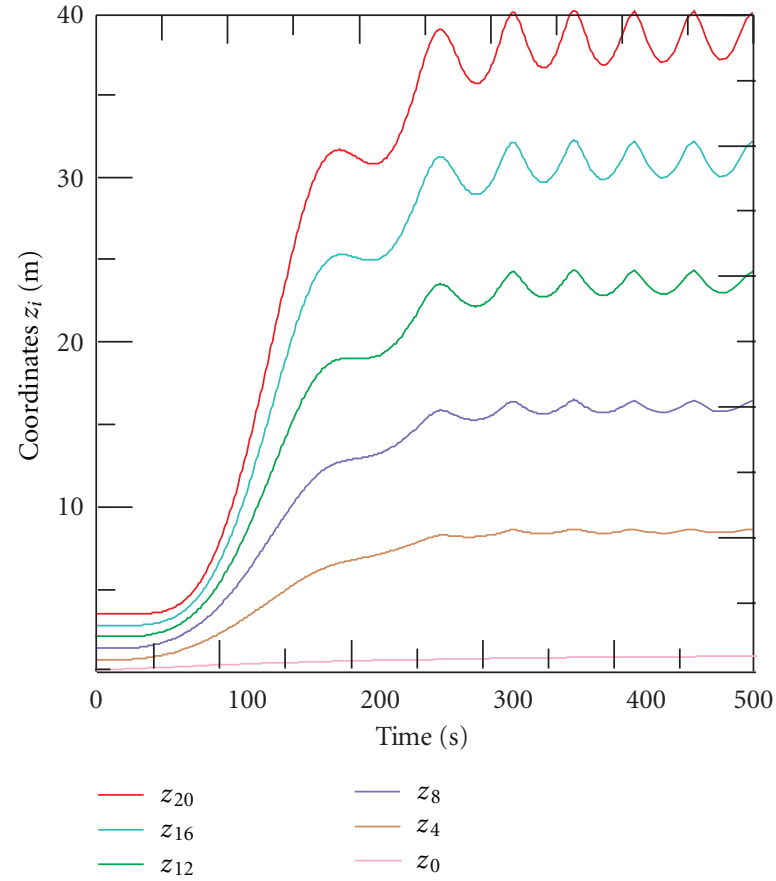

FIgURE 11: General coordinates $z_{i}$ versus time.

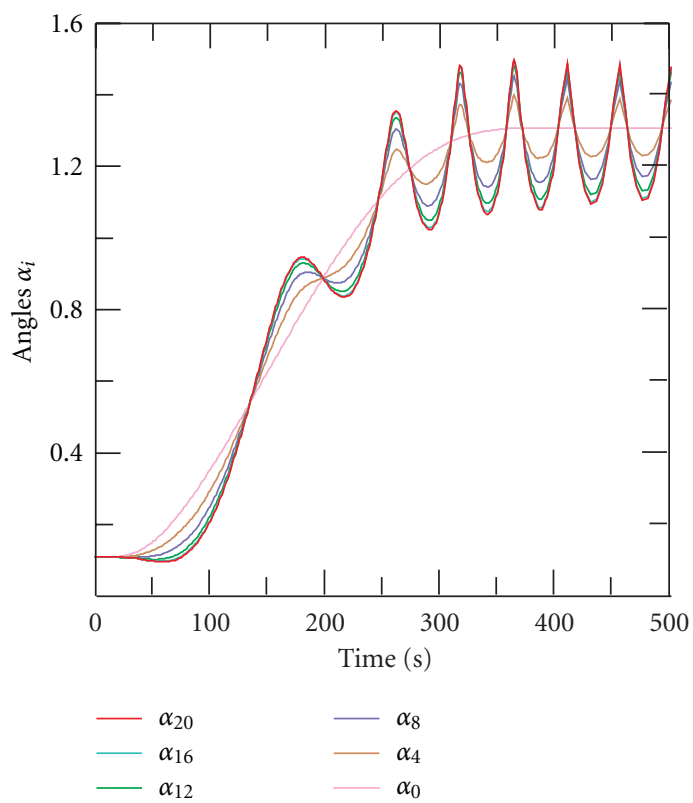

Figure 12: Time dependence of angles $\alpha_{i}$.

behaviour of the generalized co-ordinates becomes smooth enough in this case, amplitudes of oscillations of the design length do not exceed $0.15 \mathrm{~m}$. The transversal deviations have a smooth mode. The design deviates like a cantilever beam. The oscillating components are superimposed on these deviations. These deviations tend to reduction of their velocity with time more appreciable than in the previous case as duration of deployment is twice as long here. 


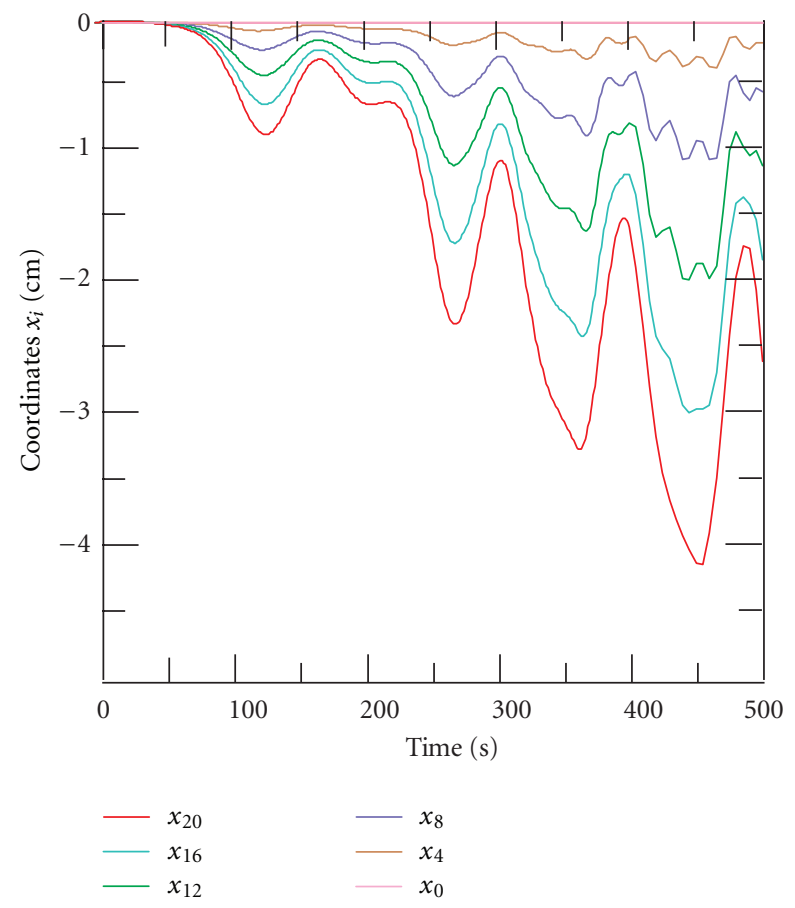

FIGURE 13: General coordinates $x_{i}$ versus time.

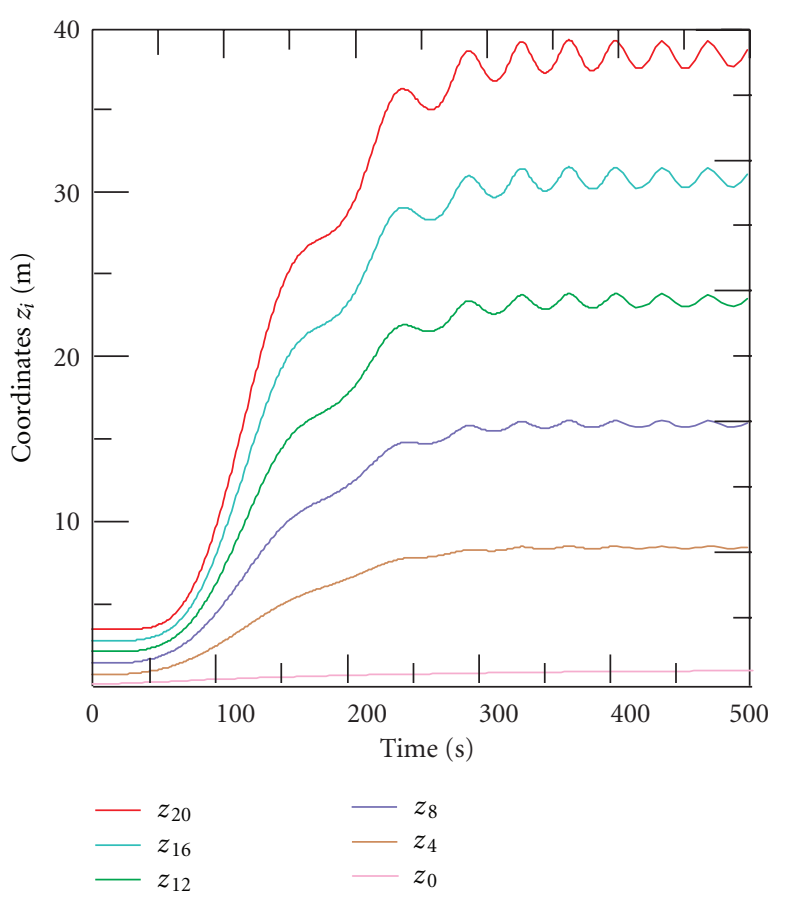

Figure 14: General coordinates $z_{i}$ versus time at optimal deployment law.

Study of SC behaviour with the five-tier pantograph on long intervals of time has shown that SC with switched-off GD under the influence of the gravitational moment enters into a condition of simple harmonic pitch oscillations. Taking into account the Coulomb friction in design, hinges have no effect on damping of SC attitude oscillations practically as

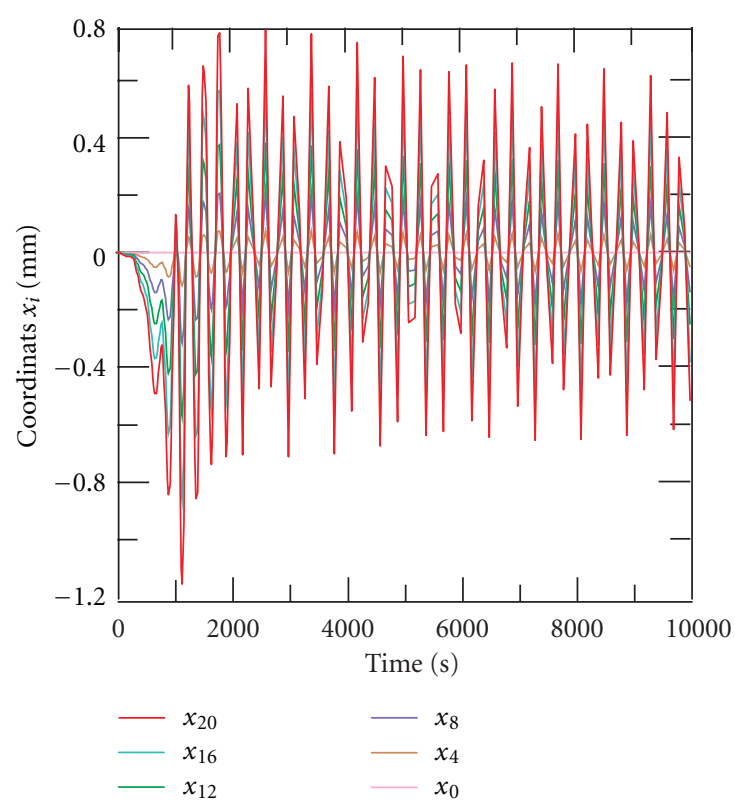

FIGURE 15: Time histories of general coordinates $x_{i}$ during and after deployment.

elastic longitudinal oscillations of the design damp quickly enough as compared with duration of deployment. Design transverse vibrations, on the contrary, damp very slowly even under the influence of forces of structural damping as their amplitudes and velocities are very small as one can see in Figure 15.

At deployment of this design from very heavy ideally stabilised space station (when the mass of the design can be neglected in comparison with the total mass of the station, and elastic oscillations have no effect on its attitude) at deployment within 100 seconds, the amplitudes and frequencies of longitudinal and transversal oscillations visibly decrease.

The analysis of the SC dynamics with the switchedon GD has shown that the pitch SC oscillations damp with sufficient for the practice speed at the accepted values of parameters of the GD system (Figure 16). The fact is noteworthy that design transverse vibrations interact with the pitch SC oscillations and damp together with them even if the structural damping is not taken into consideration (Figure 17). The behaviour of angles of GD deviation $\widetilde{\beta}_{i}$ in the process of stabilization of the pitch oscillations is shown in Figure 18.

Obtained results were compared with the case of increasing rods rigidity up to the rigid rods, the behaviour of which is described in [19]. Here asymptotic coincidence of the results was obtained. In general, the control of the total moment of momentum magnitude was realized to confirm the results. According to the known theorem in [20], $\mathbf{K}^{C}(t)=\mathbf{K}^{C}\left(t_{0}\right)+\int_{t_{0}}^{t} \mathbf{m}^{C}(\tau) d \tau$. The term in the left part is calculated on each step of evaluations as the total current moment of momentum of the system under consideration. The first term in the right part is calculated using the initial 


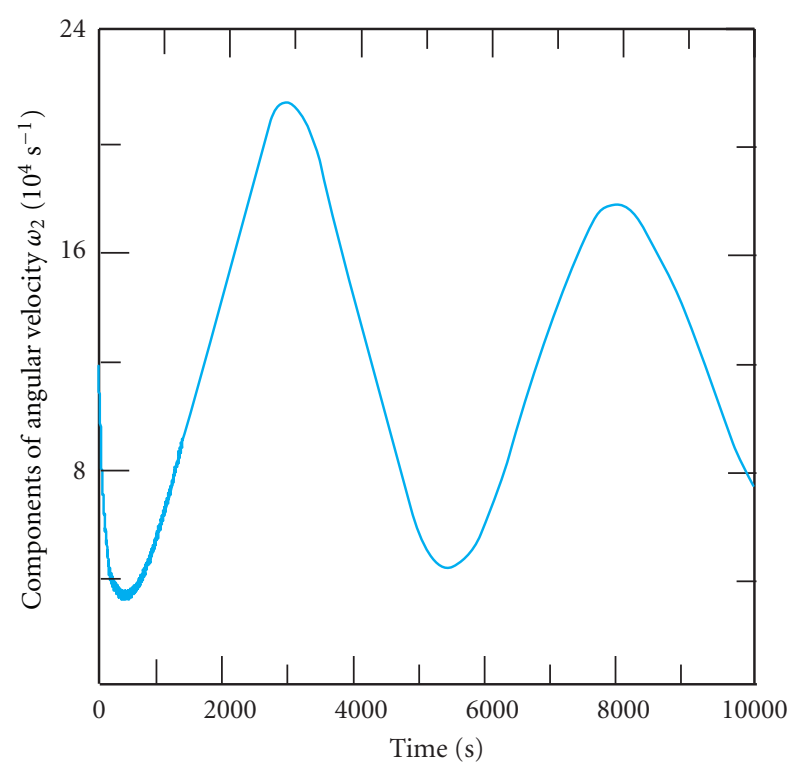

FIGURE 16: Long-term histories of $\omega_{2}$.

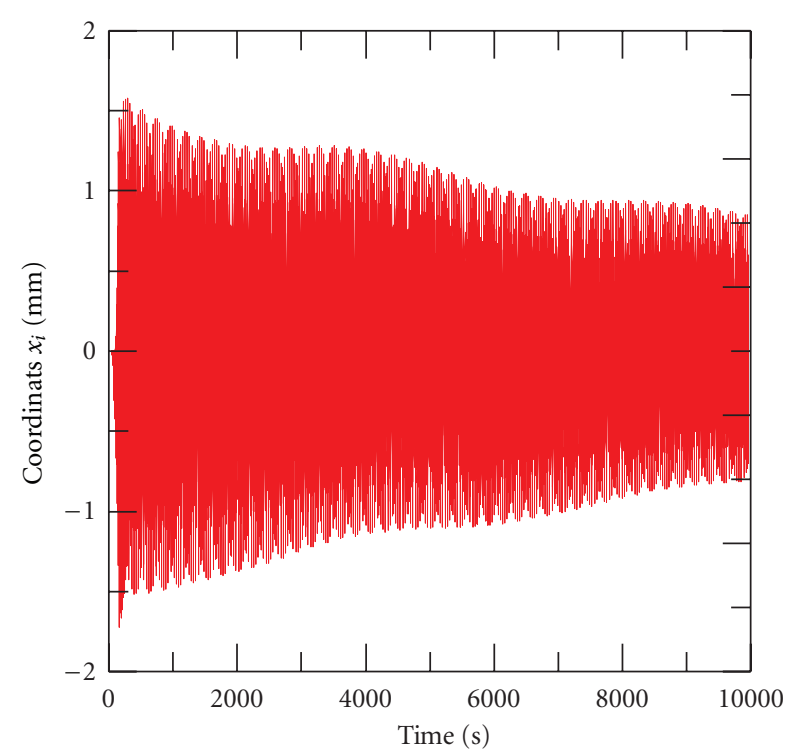

FIgURE 17: Long-term histories of general coordinates $x_{i}$.

conditions, and the second one is calculated on each step of the integrations. Good coincidence of the left and right part values indicates the absence of errors in the motion equations and in the computing program. Such a check has been included in the computing programme. During monitoring, 8 significant figures coincided for each projection, as a rule.

\section{Conclusions}

The present paper deals with the exploration of the dynamics of the gyro-gravitational stabilized spacecraft in the mode of the initial deployment of the elastic structure after the completion of the preliminary stabilization of the disordered rotation of the SC around its mass centre. Novelty of the

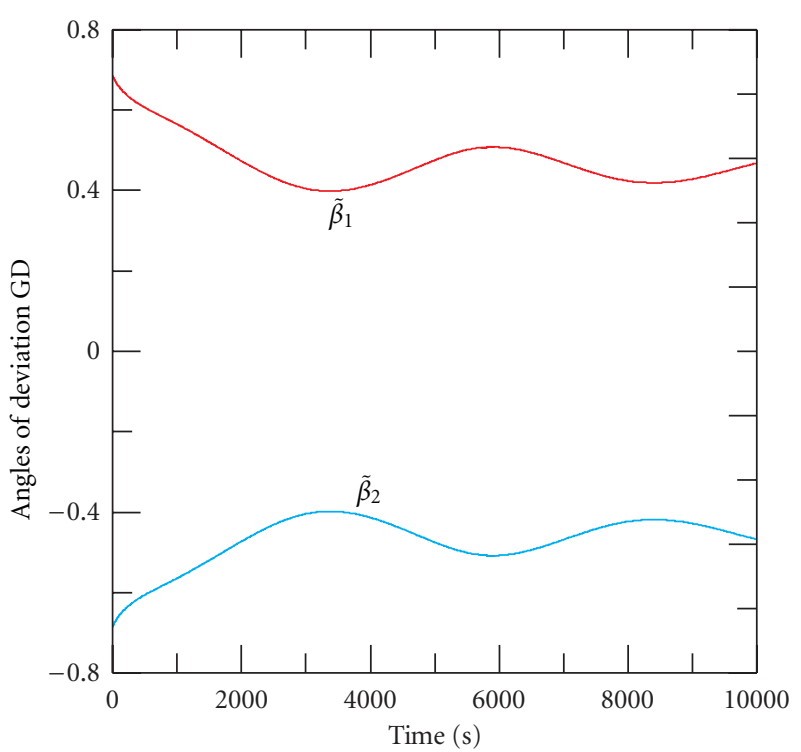

Figure 18: Angles of damping gyros deviations versus time.

approach consists in taking into account the additional internal degrees of freedom of the pantograph design in comparison with known earlier settings of the problem; in using the method of derivation of the dynamic equations of mechanical systems with internal degrees of freedom and nonstationary connections developed by author; in using optimum with respect to damping of elastic oscillations control profile for deployment of flexible designs. The mathematical model developed for this case may be regarded as the generalization of the theory of a flexible multibody system with the time-dependent configurations. The approach may be successfully extended to the modelling of the deployment dynamics of other space structures with the significant change of the configuration in the process of their functioning. The computational Fortranpackage developed for the numeric simulation and the code for Mathematica 5 developed for the symbolic calculation have general characteristics, which may be easily adopted for other deployed systems. The obtained data illustrate the method of mathematical formulation of the system dynamics with the time-dependent configuration as well as the behaviour of the SC in the process of deployment of the flexible structures. The information to be obtained in the process of the numerical simulation has allowed drawing the conclusion that deployment of the studied design may be comprehensible to practice only at a rational choice of the parameters of the SC and its deployment law. Obtained data permit to select the most appropriate deployment and gyrodampers parameters.

\section{References}

[1] W. E. Lang and G. N. Honeycutt, "Simulation of deployment dynamics of spinning spacecraft," NASA TN D-4074, 1967.

[2] G. J. Cloutier, "Dynamics of deployment of extendible booms from spinning space vehicles," Journal of Spacecraft and Rockets, vol. 5, pp. 547-552, 1968. 
[3] E. J. Bowers Jr. and C. E. Williams, "Optimization of RAE satellite boom deployment timing," Journal of Spacecraft and Rockets, vol. 7, no. 9, pp. 1057-1062, 1970.

[4] R. Barakat, "Transverse vibrations of a moving thin rod," Journal of the Acoustical Society of America, vol. 43, pp. 533$539,1968$.

[5] B. Tabarrok, C. M. Leech, and Y. I. Kim, "On the dynamics of an axially moving beam," Journal of the Franklin Institute, vol. 297, no. 3, pp. 201-220, 1974.

[6] A. K. Banerjee and T. R. Kane, "Extrusion of a beam from a rotating base," Journal of Guidance, Control, and Dynamics, vol. 12, no. 2, pp. 140-146, 1989.

[7] N. G. Creamer, "Deployment of a flexible beam from an oscillating base," Journal of Guidance, Control, and Dynamics, vol. 15 , no. 2, pp. 527-529, 1992.

[8] J. A. Lewis and E. E. Zajac, "A two-gyro, gravity-gradient satellite attitude control system," Bell System Technical Journal, vol. 43, pp. 2705-2765, 1964.

[9] D. B. Cherchas, "Dynamics of spin-stabilized satellites during extension of long flexible booms," Journal of Spacecraft and Rockets, vol. 8, no. 7, pp. 802-804, 1971.

[10] K. W. Lips and V. J. Modi, "Three-dimensional response characteristics for spacecraft with deploying flexible appendages," Journal of guidance and control, vol. 4, no. 6, pp. 650-656, 1981.

[11] K. Tsuchiya, "Dynamics of a spacecraft during extension of flexible appendages," Journal of Guidance, Control, and Dynamics, vol. 6, no. 2, pp. 100-103, 1983.

[12] K. W. Lips, W. B. Graham, F. R. Vigneron, and D. G. Hunter, "Dynamics and control characteristics for the WISP $300 \mathrm{~m}$ dipole antenna/shuttle configuration," AAS Paperno. 8, pp. 85-365, 1985.

[13] A. K. Banerjee, "Order-n formulation of extrusion of a beam with large bending and rotation," Journal of Guidance, Control, and Dynamics, vol. 15, no. 1, pp. 121-127, 1992.

[14] A. K. Banerjee and S. Nagarajan, "Efficient simulation of large overall motion of beams undergoing large deflection," Multibody System Dynamics, vol. 1, no. 1, pp. 113-126, 1997.

[15] E. M. Levin, Dynamic Analysis of Space Tether Missions, American Astronautical Society by Univelt, 2007.

[16] P. Williams, "Predictive Control of Tethered Satellite Systems, Model Predictive Control, Tao Zheng (Ed.)," InTech, 2010, http://www.intechopen.com/books/model-predictivecontrol/predictive-control-of-tethered-satellite-systems.

[17] A. E. Zakrzhevskii and V. S. Khoroshilov, "Dynamics of the orbital deployment of an elastic ring-shaped antenna," International Applied Mechanics, vol. 46, no. 6, pp. 718-729, 2010.

[18] V. I. Dranovskii, V. S. Khoroshylov, and A. E. Zakrzhevskii, "Spacecraft dynamics with regard to elastic gravitational stabilizer deployment," Acta Astronautica, vol. 64, no. 5-6, pp. 501-513, 2009.

[19] V. I. Dranovskii, A. E. Zakrzhevskii, A. P. Kovalenko, and V. S. Khoroshilov, "On the dynamics of deployment of an orbital structure with elasticelements," International Applied Mechanics, vol. 42, no. 8, pp. 959-965, 2006.

[20] A. I. Lurie, Analytical Mechanics, Springer, 2002.

[21] V. V. Beletsky, "Motion of an artificial satellite about its center of mass," Israel Program For Scientific Translations, Jerusalem NASA-TT-F-429, TT-67-51366, 1966.

[22] A. E. Zakrzhevskii, "Slewing of flexible spacecraft with minimal relative flexible acceleration," Journal of Guidance, Control, and Dynamics, vol. 31, no. 3, pp. 563-570, 2008. 

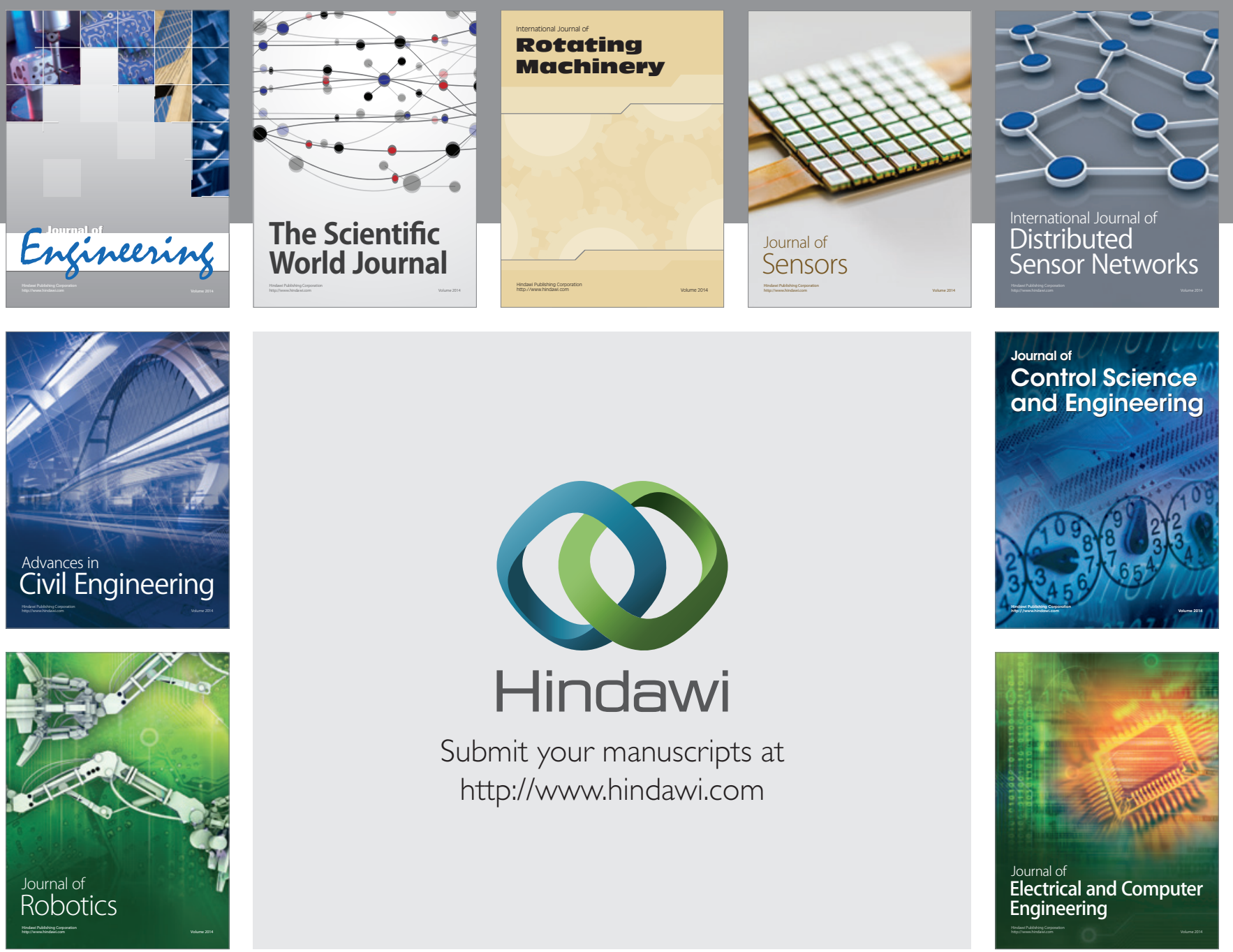

Submit your manuscripts at

http://www.hindawi.com
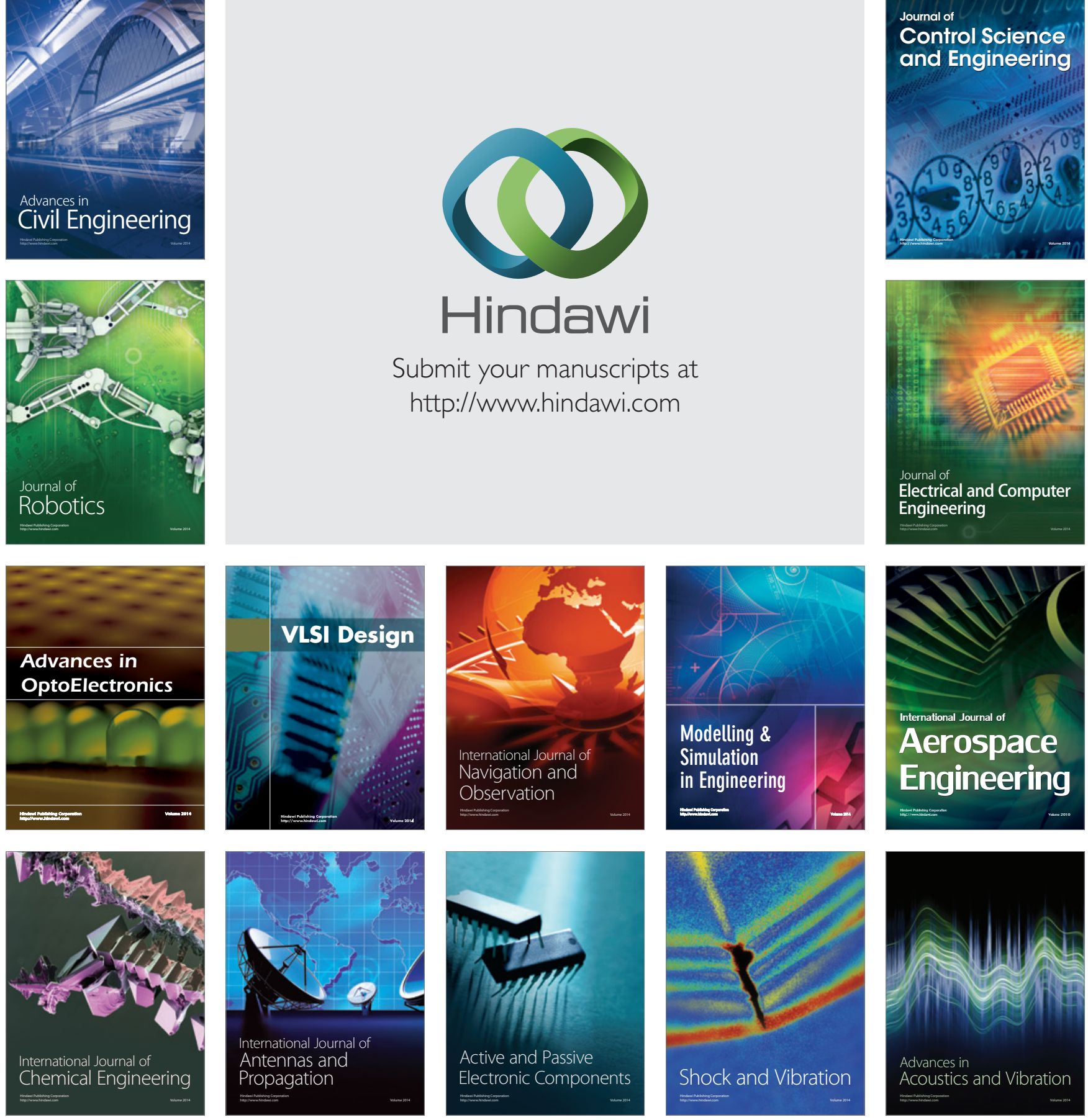